\title{
Evaluación de la Actividad y el Potencial Antioxidante de Extractos Hidro-Alcohólicos de Cúrcuma (Cúrcuma longa)
}

\author{
Armando Alvis*, Guillermo Arrazola y Walter Martinez \\ Universidad de Córdoba, Programa de Ingeniería de Alimentos, Facultad de Ingeniería, \\ Grupo de Investigación Procesos y Agroindustria de Vegetales. Carrera 6 No 76-103, Km 3, \\ vía Cereté, Córdoba-Colombia (e-mail: aalvis2@hotmail.com) \\ * Autor a quien debe dirigirse la correspondencia
}

Recibido May. 12, 2011; Aceptado Jun. 29, 2011; Versión Final recibida Sep. 01, 2011

\section{Resumen}

Se extrajeron los compuestos fenólicos del rizoma de la cúrcuma (Curcuma longa) empleando como disolvente etanol al $75 \%$. Los compuestos fenólicos presentaron una concentración de $1768.8 \mathrm{mg} / \mathrm{L}$ expresados como ácido gálico y se adicionaron a una matriz lipídica. Se determinó la actividad y el potencial antioxidante del producto natural y se comparó con el potencial antioxidante del hidrotolueno butilado (BHT), encontrándose que el extracto de cúrcuma mostró un potencial antioxidante similar al del BHT evaluado a las mismas condiciones. El estudio aporta al conocimiento sobre la extracción de compuestos antioxidantes de origen natural mostrando los beneficios que presentan frente a sus análogos de origen sintético.

\section{Evaluation of Antioxidant Activity and Potential Hydro- Alcoholic Extracts of Cúrcuma (Curcuma longa)}

\begin{abstract}
Phenolic compounds from the rhizome of turmeric (Curcuma longa) were extracted using ethanol at $75 \%$ concentration as solvent. The phenolic compounds presented a concentration of 1768.8 $\mathrm{mg} / \mathrm{L}$ expressed as gallic acid and were added to a lipid matrix. The antioxidant and potential activities of the natural product were determined and compared with the antioxidant potential of the synthetic antioxidant (BHT) finding that turmeric extracts had antioxidant potential similar to that of BHT, both tested at the same conditions. The study gives new information on the extraction of natural antioxidant compounds showing the benefits that they have over synthetic compounds.
\end{abstract}

Keywords: curcuminoids, antioxidant activity, alcoholic extracts, Cúrcuma longa 


\section{INTRODUCCION}

La cúrcuma (Cúrcuma longa) conocida también como Turmeric (Asia), es de origen asiático pertenece a la familia Zingiberaceae (Mesa et al., 2000). Se cultiva principalmente en China, India, Indonesia, Jamaica y Perú. Las propiedades del rizoma de la curcuma son muy importantes para la industria de alimentos debido al adicionarla conserva e imparte su sabor característico razón por la cual ha sido contemplado como uno de los principales ingredientes del curry (Ríos et al., 2009). Además se ha encontrado que el turmeric posee propiedades medicinales, las cuales son atribuidas principalmente al conjunto de compuestos fenólicos contenidos en dicho rizoma (curcuminoides). Estos compuestos han demostrado ser útiles para la protección frente a algunas enfermedades de tipo cancerígeno (Jayaprakasha, et al., 2005). Hoy día tiene un interés como potencial agente terapéutico para la prevención de diversas enfermedades de carácter cancerígeno (Zhou et al., 2011).

Los curcuminoides son una familia de sustancias quimiopreventivas presentes en la cúrcuma y en alimentos con extracto de cúrcuma (curry), siendo la curcumina el compuesto fenólico más estudiado. Aunque estos compuestos se conocen desde hace tiempo, ha sido en los años más recientes cuando el interés por ellas ha crecido, y lo ha hecho de forma paralela a la preocupación por los graves efectos secundarios provocados por los inhibidores sintéticos de la ciclooxigenasa2 (COX-2) y dirigido por la industria farmacéutica (Bengmark et al., 2009).

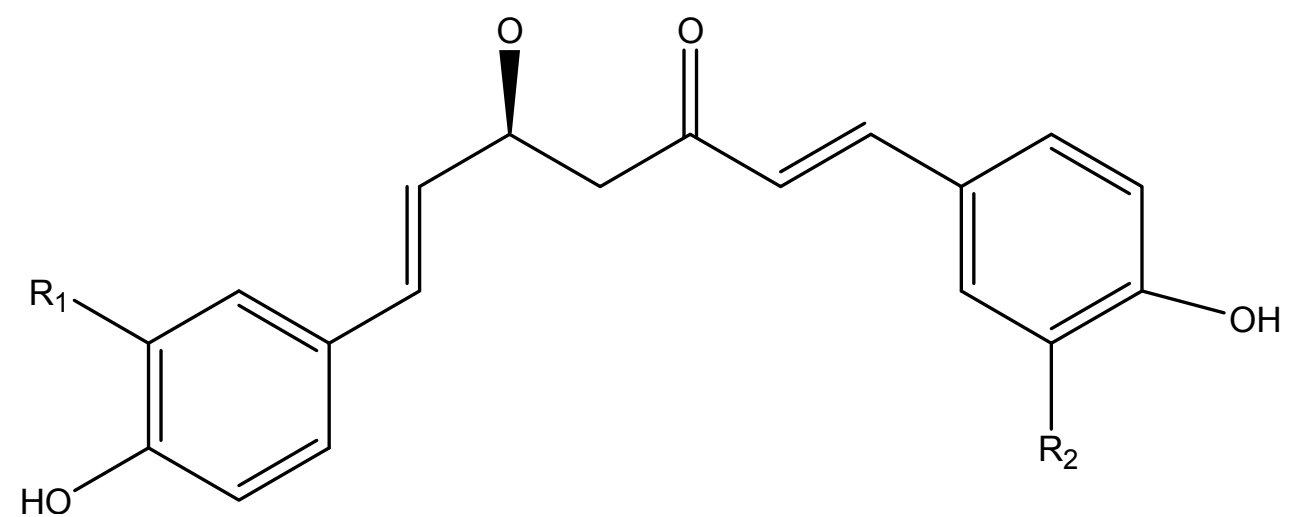

Fig. 1: Principales curcuminoides del extracto de Cúrcuma longa

\section{Curcumina}

Demetoxicurcumina

Bisdemetoxicurcumina

$\begin{array}{cc}\mathrm{R}_{1} & \mathrm{R}_{2} \\ \mathrm{OCH}_{3} & \mathrm{OCH}_{3} \\ \mathrm{H} & \mathrm{OCH}_{3} \\ \mathrm{H} & \mathrm{H}\end{array}$

La curcumina figura 1, es el principal polifenol curcuminoide encontrado en la cúrcuma, junto con otros dos compuestos de la misma naturaleza demetoxicurcumina, bisdemetoxicurcumina forman el complejo conocido como azafrán indio ó jengibre amarillo Ravindran et al., 2007, también conocida como diferuloilmetano o 1,7-bis-(4-hidroxi-3-metoxifenil)-1,6- heptadieno-3,5-diona, es un compuesto enólico de bajo peso molecular $(369.37 \mathrm{~g} / \mathrm{mol})$ con punto de fusión $183^{\circ} \mathrm{C}$, de color amarillo en medio ácido $(\mathrm{pH} 2,5-7)$ y rojo en medio básico $(\mathrm{pH}>7)$, es soluble en solventes orgánicos como dimetilsulfóxido, etanol, metanol, hexano y acetona (Casas et al., 2009; Nwoso et al., 2009).

Los compuestos fenólicos de la cúrcuma han sido centro de muchas investigaciones en el mundo por lo cual se han extraído, separados y analizados, por diferentes métodos maceración-agitación, molienda agitación, agitación extracción asistida por microondas (MWHD) (Mandal et al., 2008), cromatografía de capa delgada y columna Péret et al. (2005) y cromatografía líquida de alta resolución (Jiang et al., 2006). Ni et al. (2009), utilizando numerosos extractos, con diferentes concentraciones de solventes entre estos etanol, metanol, hexano, con el fin de encontrar sus principios, analizar sus actividades biológicas, optimizar su actividad y de explicar sus mecanismos de acción (Ravindran et al., 2007; Nwoso et al., 2009; Green et al., 2010). El objetivo 
de esta investigación fue determinar la actividad y el potencial antioxidante de extractos de cúrcuma empleando el método de oxidación acelerada (Rancimat), usando como sustrato de oxidación grasa y comparándolo con el potencial del antioxidante sintético "Butil hidroxitolueno (E321)".

\section{MATERIALES Y METODOS}

Los rizomas de cúrcuma (Curcuma longa) se recolectaron aleatoriamente de cultivos de plantas aromáticas de la ciudad de Montería (Colombia), ésta se realizó en los meses de septiembre y octubre de 2009. Las muestras fueron secadas a $50 \pm 1{ }^{\circ} \mathrm{C}$ en estufa Memmert, modelo U400, con circulación forzada. Posteriormente, los rizomas de la cúrcuma fueron homogenizados empleando un molino de cuchillas de alta velocidad Grindomix, modelo GM200 (tamaño de partícula máximo de $0.5 \mathrm{~mm}$. Las muestras molidas y secas fueron almacenadas en una desecadora durante la realización de los ensayos programados.

Influencia del grado alcohólico: Los extractos fueron acondicionados por agitación a 1200 r.p.m en un equipo FISCHER ISO TEMP, modelo 11-102-49SH. Se tomaron 1,0 $\pm 0,1 \mathrm{~g}$ de las muestras molidas y secas con $20 \mathrm{~mL}$ de disolvente (mezcla de etanol/agua) en tubos centrífugos con tapas rosca durante una hora. Con el fin de determinar la concentración alcohólica adecuada, se varió la concentración de etanol en la mezcla hidro-alcohólica desde cero (0) (agua pura) hasta $100 \%$ (equivalente a etanol al $95 \%$ ). El contenido de fenoles se determinó siguiendo el procedimiento descrito por Slinkard y Singleton (1977) empleando el reactivo de Folin-Ciocalteu.

Capacidad antioxidante: Determinada la concentración alcohólica más apropiada para la extracción de los compuestos fenólicos de la cúrcuma, se procedió a la obtención de los correspondientes extractos. Los mismos fueron convenientemente diluidos e inmediatamente analizados por duplicado para determinar la capacidad antioxidante mediante los ensayos FRAP y ABTS. El ensayo FRAP (Ferric Reducing Activity Power se realizó de acuerdo con el procedimiento propuesto por (Benzie y Strain, 1996). El mismo consistió en añadir $1500 \mu \mathrm{L}$ del reactivo FRAP recién preparado a $50 \mu \mathrm{L}$ de extracto en un tubo de ensayo. La reacción se lleva a efecto a $37{ }^{\circ} \mathrm{C}$ y se lee absorbancia a $593 \mathrm{~nm}$, luego de transcurrido 30 minutos. La curva de calibración de $\mathrm{Fe}^{2+}$ se hizo preparando soluciones de concentración conocida en el intervalo de 200 a $1500 \mu \mathrm{M}$ de $\mathrm{Fe}^{2+}$ en medio acuoso, empleando la sal de Mohr [Fe( $\left.\left(\mathrm{NH}_{4}\right)_{2} \mathrm{SO}_{4} \cdot 6 \mathrm{H}_{2} \mathrm{O}\right]$.

Ensayo con ABTS: Se siguió el método propuesto por Re et al. (1999), controlando 10 minutos como tiempo final de la reacción de decoloración del radical ABTS (2,2'azinobis-(3etilbenzotiazolin 6-ácido sulfónico)) Un (1) $\mathrm{mL}$ de la solución diluida de radical ABTS (con absorbancia $1,00 \pm 0,02 \mathrm{UA}$ ) se añade a un tubo de ensayo que contiene $50 \mu \mathrm{L}$ de extracto y a otro conteniendo $50 \mu \mathrm{L}$ del disolvente empleado tomándolo como referencia. Una vez transcurrido los diez minutos se lee la absorbancia de ambos a $734 \mathrm{~nm}$. La curva de calibración se preparó tomando como sustancia de referencia el Trolox, (ácido 6-hidroxi-2,5,7,8-tetrametil-2cromanocarboxílico) considerado análogo de la vitamina $\mathrm{E}$, en concentraciones entre 0 y $7 \mu \mathrm{M}$. La variación de las réplicas analíticas siempre fueron $<10 \%$ para ambos ensayos, en caso contrario, los análisis fueron repetidos para confirmar el valor.

Potencial antioxidante: El ensayo se hizo en un equipo RANCIMAT modelo 743 (Metrohm, Suiza), se empleó como matriz oxidante grasa sin adición alguna de antioxidante. La temperatura de reacción fue $110^{\circ} \mathrm{C}$ y el flujo de aire de $20 \mathrm{~L} \cdot \mathrm{h}^{-1}$. Cantidades del extracto etanolico previamente calculadas, fueron añadidas a los tubos de reacción conteniendo $3,0 \mathrm{~g}$ de grasa, a fin de tener una concentración final de compuestos fenólicos entre 0 y $100 \mathrm{mg} \cdot \mathrm{kg}^{1}$, para el extracto de cúrcuma y para el BHT. La relación entre los tiempos de inducción de la grasa control y la grasa conteniendo antioxidantes fue la base para la evaluación del potencial antioxidante, ecuación 1.

Potencial antioxidante $=\frac{\text { Tiempo de inducción grasa estabilizante }}{\text { Tiempo de inducción grasa control }}$ 
En la determinación de la concentración alcohólica más apropiada para la extracción de los compuestos fenólicos en cúrcuma el factor fue: la concentración alcohólica en cinco niveles (100\% agua), (25:75 etanol /agua), (50:50 etanol /agua), (75:25 etanol /agua), ( $95 \%$ etanol), y para el potencial antioxidante el factor fue la concentración y el tipo de antioxidante tres repeticiones para cada tratamiento.

\section{RESULTADOS Y DISCUSIÓN}

Los resultados en cuanto a la selección del mejor disolvente para la extracción de los compuesto fenólicos (CF) se muestran en la figura 2. La concentración alcohólica ejerce efecto significativo $(\operatorname{Pr} \leq 0,05)$ en la extracción de los compuestos fenólicos contenidos en el rizoma de la cúrcuma.

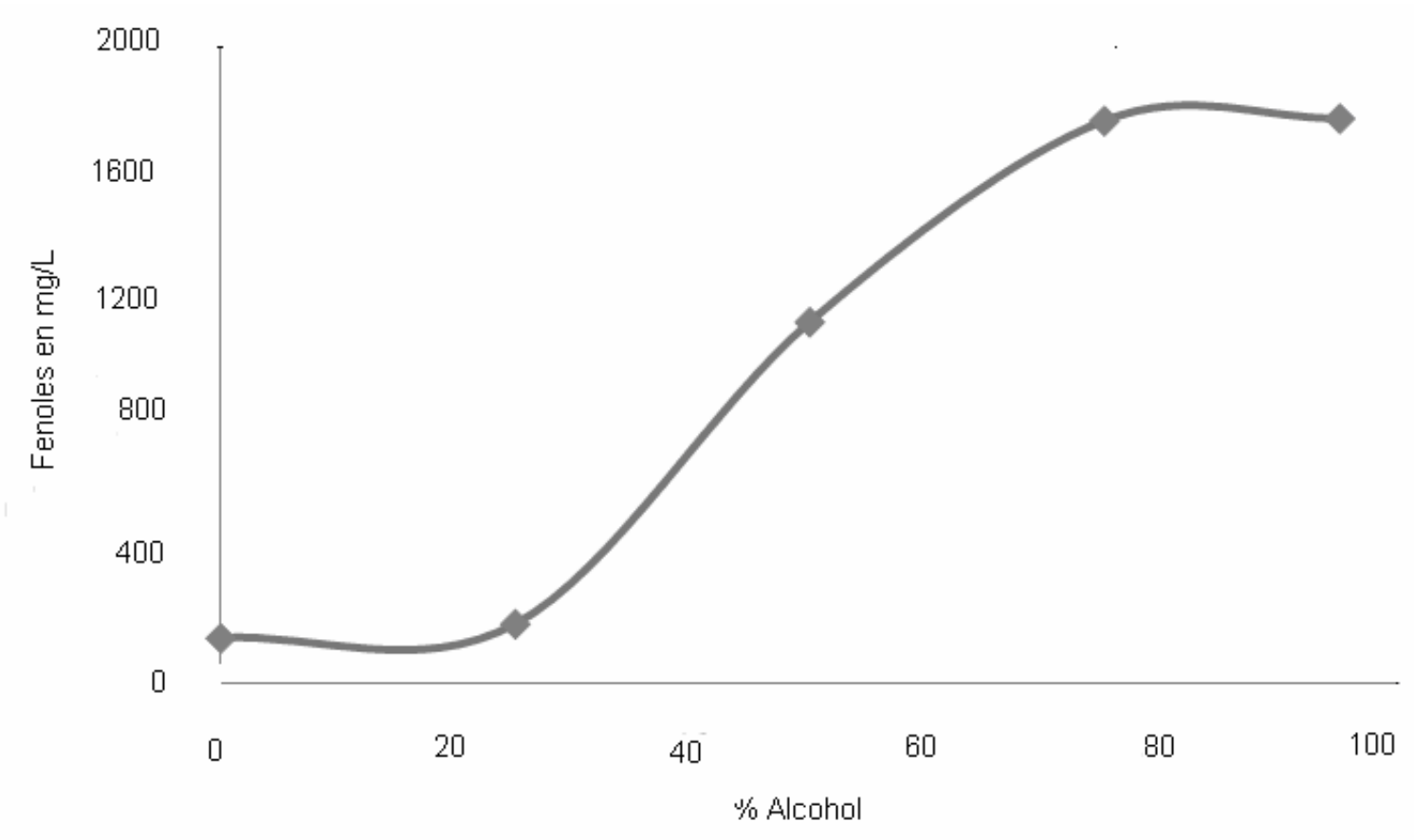

Fig. 2: Influencia del grado alcohólico en la extracción de los compuestos fenólicos (CF) en (Cúrcuma longa)

La figura 2 muestra que el incremento del grado alcohólico mejora la extracción de los compuestos fenólicos, es decir, en la medida en que se aumenta la concentración etanólica se extrae una mayor masa de compuestos fenólicos. Para las concentraciones etanólicas de 75 y $95 \%$, no existió diferencia significativa en la extracción de los compuestos fenólicos de la cúrcuma, es decir la masa de compuestos fenólicos extraída a estas dos concentraciones era la misma, lo cual indica que se puede extraer con cualquiera de estas dos concentraciones. Para los ensayos posteriores se extrajeron empleando un concentración alcohólica de $75 \%$ V/V.

El fenómeno anterior se puede atribuir a la polaridad de las soluciones empleadas para la extracción de los CF, lo cual indica que los CF tienen una polaridad al etanol que al agua (Ríos et al., 2009). Según Bengmark et al. (2009), los compuestos fenólicos mayoritarios presentes en los rizomas de la cúrcuma, son los curcuminoides (figura 1). Estos compuestos al estar conformados por un elevado número de carbonos tienen mayor polaridad al etanol puro que al agua, (Centeno et al., 2004). La caracterización del extracto en cuanto a su actividad se muestra en la tabla 1. Los valores de fenoles, ABTS y FRAP corresponden a la media y entre paréntesis la desviación estándar $(\mathrm{n}=3) .{ }^{2} \mathrm{mg}$ ácido gálico/L; ${ }^{3} \mathrm{mg}$ de Trolox/L; ${ }^{4} \mu$ moles $\mathrm{Fe}^{2+} / \mathrm{L}$. 
Tabla 1. Caracterización del extracto de Cúrcuma en cuanto al contenido fenólico y su actividad antioxidante.

\begin{tabular}{|c|l|c|c|}
\hline Extracto & \multicolumn{1}{|c|}{ Fenoles mg/L ${ }^{2}$} & ABTS $^{3}$ & FRAP $^{4}$ \\
\hline Cúrcuma & $1768,8(5,5)$ & $2649(6.8)$ & $17603,8(20.8)$ \\
\hline
\end{tabular}

La Tabla 1 muestra que la cúrcuma posee un elevado contenido de compuestos fenólicos (CF), el cual está por encima de otras plantas aromáticas (hierbabuena, menta, perejil, albahaca común y orégano francés, las cuales tienen un contenido fenólico de $58.7 \mathrm{mg} / 100,12.3 \mathrm{mg} / 100,30.4$ $\mathrm{mg} / 100,8.4 \mathrm{mg} / 100$ gramos de muestra, respectivamente (Rodríguez et al., 2006). De igual forma se observa que el extracto posee una alta capacidad antioxidante y un alto poder secuestrante de radícales libres.

Surveswaran et al. (2007), extrajo los compuestos fenólicos con metanol al $80 \%$ e hizo la caracterización del extracto empleando los mismos métodos que se hicieron en esta investigación, sin embargo, obtuvo valores por debajo a los reportados en este trabajo lo cual puede atribuírsele a la polaridad del solvente empleado y lo que pone de manifiesto que los compuestos fenólicos de la cúrcuma tienen una mayor afinidad a los solventes apolares.

Potencial antioxidante: La concentración de los antioxidantes tiene efecto significativo $(\operatorname{Pr} \leq 0,05)$ en la inhibición de la oxidación de la matriz oxidante. Sin embargo, no existe diferencia significativa $(\operatorname{Pr} \leq 0,05)$ entre los antioxidantes evaluados. Por lo cual se puede decir que el extracto de cúrcuma tiene un potencial antioxidante similar al del BHT. Lo que pone de manifestó el alto potencial antioxidante de dicho extracto evaluados a las mismas condiciones. En la figura 3 se observa que en la medida en que se aumenta la concentración del antioxidante, aumentan el factor protector que ejerce el antioxidante en la matriz grasa.

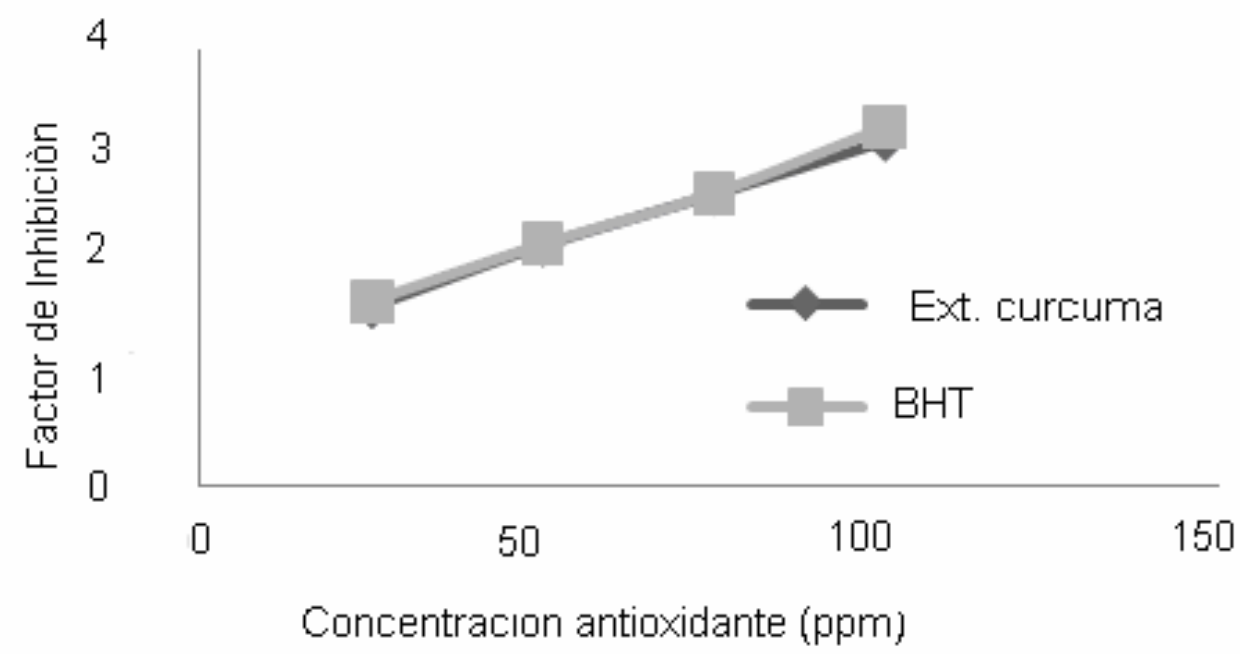

Fig. 3: Inhibición de la oxidación del extracto de cúrcuma y el BHT en grasa de cerdo.

Kulisic et al. (2005), reportaron un factor de inhibición de oxidación de la grasa de cerdo de 2,0; y 1,7 usando como antioxidante aceite esencial de otras plantas aromáticas entre estas Origanum vulgare $L$ y Thymus vulgaris $L$. respectivamente, sin embargo, a pesar de emplear concentraciones más elevadas y condiciones menos drásticas a las empleadas en esta investigación obtuvieron menores índices de inhibición, lo que pone de manifiesto el elevado potencial antioxidante del extracto de cúrcuma. 


\section{CONCLUSIÓN}

La concentración alcohólica óptima para la extracción de los compuestos fenólicos fue de $75 \%$. El extracto de cúrcuma obtenido a dicha concentración alcohólica presentó elevado contenido de compuestos fenólicos, compuesto antioxidantes y poder secuestrante de radicales libres. El extracto alcohólico de cúrcuma posee un potencial antioxidante similar al que ejerce el $\mathrm{BHT}$ en la inhibición de la oxidación para una matriz lipidica, evaluados a las mismas condiciones.

\section{AGRADECIMIENTOS}

Los autores expresan su total agradecimiento a la Universidad de Córdoba, al grupo de Investigación Procesos y Agroindustria de Vegetales, al Codecyt y al Instituto de Investigaciones para la Industria Alimentaria (IIIA).

\section{REFERENCIAS}

Bengmark, S., M. Mesa y A. Gil, Efectos saludables de la cúrcuma y de los curcuminoides, Nutrición Hospitalaria 24(3):264-272 (2009).

Benzie, I. y J. Strain, The ferric reducing ability of plasma (FRAP) as a measure of antioxidant power. the FRAP assay. Anal biochemestry 239(2), 70-76 (1996).

Casas, J., H. Castillo, J. Noy, A. Palomares y R. Rodríguez, Elaboración de papel indicador a base de extractos naturales: una alternativa fundamentada en experiencias de laboratorio para el aprendizaje del Concepto de pH, Revista Eureka Enseñ. Divul. Cien., 9(2):302-314 (2009).

Centeno, G., F. Trejo, J. Ancheyta y A. Carlos; Precipitación de asfáltenos del crudo de maya en un sistema de a presión, Journal of the Mexican, 179-188 (2004).

Green, C. E., y otros 5 autores, Extraction, processing, and storage effects on curcuminoids and oleoresin yields from Curcuma longa L. grown in Jamaica. Journal of Agricultural and Food Chemistry, 56, 3664-3670. (2008).

Jayaprakasha, K., L. Rao y K. Sakariah, Chemistry and biological activities of C. Longa. Trends in Food Science and Technology 16 (12): 533-548 (2005).

Jiang, H., B. Timmermann y D. Gang, Use of liquid chromatography-electrospray ionization tandem mass spectrometry to identify diarylheptanoids in turmeric (Curcuma longa L.) rhizome. Journal of Chromatography A. 1111 (1): 21-31 (2006).

Kulisic, T., A. Radonic y M. Milos, Inhibition of lard by fractions of different essential oils. Revista de grasas y aceites 56(4): 284-291(2005).

Mandal, V., Y. Mohan y S. Hemalatha, Microwave assisted extraction of curcumin by samplesolvent dual heating mechanism using Taguchi L9 orthogonal design. Journal of Pharmaceutical and Biochemical Analysis 46 (2): 322- 327 (2008).

Mesa, D., M. Ramírez, M. Aguilera, A. Ramírez y A. Gil, Pharmacological and nutritional effects of curcuma longa I. Extracts and pplying ides, Ars Pharmaceutica, 41(3):307-321(2000).

Ni, H., Zhou, X. H., Li, H. H., \& Huang, W. F. Column chromatographic extraction and preparation of cordycepin from Cordyceps militaris waster medium. Journal of Chromatography B, 877, 21352141.(2009).

Nwoso, S., O. Adaramoye y E. Ajaiyeoba, Oral administration of extract Curcuma longa lowers Blood glucose and attenuates alloxan-induced Hyperlipidemiain diabetic rabbits, Journal of Nutrition 8 (5):625-628 (2009). 
Péret, L., A. Cherubino, R. Alves, L. Dufossé y M. Glória, Separation and determination of the physicochemical characteristics of curcumin, demethoxycurcumin and bisdemethoxycurcumin. Food Research International, 38 (8): 1029-1044 (2005).

Ravindran, P., K. Nirmal y K. Sivaraman, Turmeric the genus Curcuma. Taylor y Francis Group, New York, p 47-453 (2007).

Re, R. N. Pellegrini, A. Proteggente, A. Pannala, M. Yang y C. Rice-Evans, Antioxidant activity pplying an improved ABTS radical cation decolorization assay. Free Radic. Biol. Med., 1231-1237 (1999).

Ríos, E., A. Duque y F. León, Spectroscopy and chromatography characterization of curcumin extracted from the rhizome of turmeric crops in the department of Quindío (Colombia), Revista Investigación Universidad del Quindío 19(1):18-22 (2009).

Rodríguez, J., O. Valdez y A. Alemán, Evaluación de la actividad antioxidante de cinco hierbas aromáticas. Revista Ciencia y Tecnología de Alimentos 16(1):30-36 (2006).

Slinkard, K. y V. Singleton; Total Phenol Analysis: Automation and Comparison with Manual Methods, Journal Enol. Vitic. 28(1):49-55 (1977).

Surveswaran, S., Y. Cai, H. Corke, y M. Sun, Systematic evaluation of natural phenolic antioxidants from 133 Indian medicinal plants. Food Chemistry 102, 938-953 (2007).

Zhou, H., Beevers, C. S., \& Huang, S. The targets of curcumin. Current Drug Targets, 12(3), 332347. (2011). 
